\title{
Holography as Cutoff: a proposal for measure of inflationary universes
}

\author{
Fábio Novaesa and and Bruno Carneiro da Cunhab \\ Departamento de Física, Universidade Federal de Pernambuco, \\ 53901-970, Recife, Pernambuco, Brazil
}

\begin{abstract}
We propose the holographic principle as a dynamical cutoff for any quantum theory of gravity, incorporating ideas of effective field theory. This is done by viewing the holographic bound as a limit on the number of degrees of freedom that can be turned on before the geometrical picture of gravity loses applicability. We illustrate the proposal by revisiting the problem of defining a measure for homogeneous and isotropic spacetimes coupled to a scalar field and conclude by discussing the implications to the single scalar field inflationary model.
\end{abstract}

Keywords: Inflation, Holography, Cosmology

\footnotetext{
a fabiomnsantos@df.ufpe.br

b bcunha@df.ufpe.br
} 


\section{INTRODUCTION}

The concilliation between Quantum Mechanics and gravity, in the guise of General Relativity, is the most important open problem of theoretical physics. One of the difficulties can be attributed to the fact that the Einstein-Hilbert lagrangian is non-renormalizable. As classical (or effective) theories, non-renormalizable field theories are fine, but as quantum theories they are heavily dependent on the cutoff, and a cutoff scheme that respects the considerable symmetries of General Relativity is still lacking. If such scheme were to be found, we would be effectively dealing with a quantum system of a finite number of degrees of freedom and no such obstacle to the definition of a quantum version of General Relativity would exist.

At any rate, this problem has been somewhat circumvented in the last two decades: in a very deep sense, one should not think of General Relativity as a fundamental theory. The effective microscopic degrees of freedom are not geometric, and the metric only arises as an effective, macroscopical, description. Trying to quantize the space of metrics directly is not only ineffective, it is also not a true description of quantum gravity.

On the other hand, many aspects of semi-classical gravity can be well modelled by General Relativity: the laws of Black Hole Thermodynamics and several descriptions of quantum effects of fields in curved backgrounds rely heavily on the geometrical interpretation of quantum gravity. In this sense, the microscopic description doesn't help us gain insight on the geometrical character of many quantities. The epitome of such quantities being the Bekenstein-Hawking entropy.

The number of degrees of freedom available as area is perhaps the most impressive result of semi-classical gravity. Not only it applies to all macroscopical black-holes, but also for cosmological settings [1-3] $]$. The universality of the result has a multitude of heuristic explanations, but so far the microcanonical descriptions are non-geometrical in nature. This lack of intuition about the result led several proposals to promote it to a fundamental principle behind gravity itself.

In this letter we will attempt to operationalize the notion of quantum gravity by using a truncated version of General Relativity as a quantum theory, and the Holographic Principle

as a means of defining a natural cutoff of the theory. We will illustrate the procedure considering the measure of initial conditions for an inflationary universe and spend some 
time on the problem of naturalness.

\section{HOLOGRAPHY}

The idea that the number of degrees of freedom in some region of space is bounded by the area of its boundary is a natural consequence of the laws of Thermodynamics of Black Holes. In its essence, the argument is deceptively simple: in the absence of any other forces

of nature, the natural endpoint for the evolution of any system is a black-hole. As the second law of thermodynamics tells us that in such evolution the entropy cannot decrease, then it should reach its maximum value for a black hole.

Upon closer inspection, however, the complications begin to unfold. The interpretation of entropy as a measure of the number of degrees of freedom available to the system leaves us at first with the confusing task of recognizing which degrees of freedom of the system the Bekenstein-Hawking entropy is counting. Again, a heuristic argument hints at the solution: imagine a gas of free particles with temperature $T$, interacting only gravitationally. As there is no force counteracting gravity, the system will undergo gravitational collapse for generic initial conditions. During the process, as the gas becomes denser and denser, an observer sitting far away from the gas actually measures the temperature of the gas reducing to the Black Hole temperature.

We can draw two conclusions from this example: i) the Bekenstein-Hawking temperature is assigned to degrees of freedom other than the kinetic or the (other) potentials between the system; and ii) The Bekenstein-Hawking entropy is so much larger than the usual values found in non-gravitational systems that in the end it acts as a "heat bath", such that the non-gravitational degrees of freedom thermalize with the Black Hole temperature. Were it the other way around, having a black hole at the end of the process would violate the second law of thermodynamics for a large class of initial conditions.

The question that can be posed now is what happens with the description of the system as the "non-gravitational" entropy increases and surpasses the "gravitational" entropy? From the point of view of general relativity, such system is unstable, exactly like a supercooled or superheated system. The dynamics of the system is no longer given by General Relativity since the "thermodynamical" variables like the metric are no longer well defined. The description of the end result is to be achieved with a microscopic theory, and even though 
one can still make predictions on general grounds, like Hawking radiation - or the Maxwell construction of the van der Waals fluid - the fate of the process relies ultimately on this underlying theory. Questions like unitarity of evolution and presumed information loss can only be answered there.

One can draw from the generic conclusions summing up the arguments above: i) the Bekenstein-Hawking entropy counts the number of degrees of freedom of gravity itself, and ii) The entropy can also be seen as a bound on the number of degrees of freedom of gravity that have geometrical interpretation. In other words, it can be seen as a cutoff on the number of degrees of freedom of classical gravity.

Since the nature of this cutoff is very different from the usual maximum momentum value, let us try then to make the idea a little more concrete. Consider a globally hyperbolic spacetime, i.e., with a global time function such that its topology is $\mathbb{R} \times \Sigma$, with $\Sigma$ being connected. Let $a$ be the "radius of the universe", that is, an observable of $\Sigma$ which serves as the scale factor. We will label a state $|a\rangle$ defined on the Hilbert space of gravity with definite value for the operator $\hat{a}$. The proposal can be made, explicitly, by defining the maximum number of non-geometrical degrees of freedom $\mathcal{N}(a)$ associated with the aforementioned states as:

$$
\mathcal{N}(a)=\sum_{i}\langle a,\{i\} \mid a,\{i\}\rangle=\frac{1}{4}\left(\frac{a}{\ell_{P}}\right)^{D-2} \frac{2 \pi^{(D-1) / 2}}{\Gamma((D-1) / 2)},
$$

the last fraction being the area of the unit sphere. The Planck length is $\ell_{P}$. The index of summation $\{i\}$ refer to states which share the same value for $a$. These may refer to "matter" anisotropies, or some other type of internal degree of freedom. We are of course supposing that the number of degrees of freedom and the area can be independently measured. If the area cannot be measured independently of the scale factor $a$, then the functional form above can be better justified without "quantum corrections". At any rate, higher order corrections to the entropy will not change the discussion.

This cutoff shows up as a natural regulator of the purposed quantum theory of gravity. Rather than being an inconsequential device to keep amplitudes finite, this serves as a fundamental parameter of the theory, in the sense of the role of the cutoff in an effective field theory.

The usefulness of (11) as a cutoff is somewhat impaired in the general case, since $a$ is a non-local function on $\Sigma$, presumably also a non-local function of the degrees of freedom, 
assuming that there are local degrees of freedom that are also gauge invariant. However, there is a way of decoupling $a$ in a generic class of metrics, still wide enough to be of interest. We will formulate this in the following.

\section{INITIAL DATA AND INFLATIONARY EVOLUTION}

The proposal for the cutoff is not entirely technical, though. To illustrate the argument, let us define the scale factor in a more geometrical fashion, and review the role of the Hamiltonian formalism of General Relativity. To start, consider metrics with zero lapse vector:

$$
d s^{2}=-N^{2} d t^{2}+h_{i j} d x^{i} d x^{j}
$$

The hamiltonian formulation of gravity essentially treats $h_{i j}$ as dynamical variables. Their conjugate momenta have an interesting interpretation. Let us define the extrisinc curvature:

$$
K_{a b}=\frac{1}{2} \mathscr{L}_{n} h_{a b}=\frac{1}{2 N} \dot{h}_{a b}
$$

where $n^{a}$ is the unit vector in the time direction. By simple algebra, we can relate $K_{a b}$ to the covariant derivative of $n^{a}$, given that $h_{a b}=g_{a b}+n_{a} n_{b}$ :

$$
\begin{aligned}
K_{a b} & =\frac{1}{2}\left[n^{c} \nabla_{c} h_{a b}+h_{c b} \nabla_{a} n^{c}+h_{a c} \nabla_{b} n^{c}\right] \\
& =\left(n^{c} \nabla_{c} n_{(a}\right) n_{b)}+\nabla_{(a} n_{b)}=h_{(a}^{c} h_{b)}^{d} \nabla_{c} n_{d}
\end{aligned}
$$

i.e., the projection of the covariant derivative of $n^{a}$ to the spacial slice. Writing the curvature scalar in terms of the extrisinc curvature we have (see, for instance, (E.2.29) in [4]):

$$
{ }^{D} R={ }^{(D-1)} R-K^{2}+K_{a b} K^{a b},
$$

where ${ }^{(D-1)} R$ is the curvature scalar associated with $h_{a b}$. The conjugate momentum to $h_{a b}$ can be now computed, using the volume element $\sqrt{-g}=N \sqrt{h}$ :

$$
\pi^{a b}=\frac{\partial S_{\mathrm{EH}}}{\partial h_{a b}}=\frac{\partial \mathcal{L}}{\partial \dot{h}_{a b}}=\sqrt{h}\left(K^{a b}-K h^{a b}\right) .
$$


And, by a Legendre transformation, one computes the Hamiltonian density:

$$
\mathcal{H}=\pi^{a b} \dot{h}_{a b}-\mathcal{L}=-\sqrt{h} N\left[{ }^{(D-1)} R-h^{-1}\left(\pi^{a b} \pi_{a b}-\frac{1}{D-2} \pi^{2}\right)+\mathcal{H}_{\text {matter }}\right]
$$

For a scalar field, for instance, $\mathcal{H}_{\text {matter }}=1 / 2\left[(D \phi)^{2}+h^{-1} \Pi^{2}+V(\phi)\right]$, where $\Pi=\sqrt{h} N^{-1} \dot{\phi}$ is the momentum conjugate to $\phi$. Let us keep the expression generic for the time being.

Now assume that the only relevant term to the evolution is the expansion of the metric. $K_{a b}$ (and then $\pi^{a b}$ ) is then related to the expansion factor:

$$
K_{a b}=\frac{1}{N} \theta h_{a b} \quad \therefore \quad \pi^{a b}=-\frac{D-2}{N} \theta \sqrt{h} h^{a b} .
$$

More importantly, the natural variable becomes the volume element $\sqrt{h}$, to which we find the conjugate momentum:

$$
\frac{\partial S_{\mathrm{EH}}}{\partial \sqrt{h}}=\frac{\partial S_{\mathrm{EH}}}{\partial h_{a b}} \frac{\partial h_{a b}}{\partial \sqrt{h}}=\frac{2}{\sqrt{h}} h_{a b} \frac{\partial S_{\mathrm{EH}}}{\partial h_{a b}}=\frac{2}{\sqrt{h}} \pi
$$

With these formulas we can write the constraint $\mathcal{H}=0$ in terms of $\sqrt{h}$ and the matter fields, in a Hamilton-Jacobi form:

$$
{ }^{(D-1)} R+\frac{1}{4(D-1)(D-2)}\left(\frac{\partial S_{\mathrm{EH}}}{\partial \sqrt{h}}\right)^{2}+\mathcal{H}_{\text {matter }}=0,
$$

For the type of metrics in which $K_{a b} \propto h_{a b}$, one can define the scale factor as $\sqrt{h}=a^{D-1}$ and then $\theta$ in (8) is essentially the expansion factor $\dot{a} / a$. The expansion factor is then the momentum conjugate to the scale factor, and (10) is nothing more than (half) of the FRW equations written in a fancier language.

The equation (10) encodes the dynamics of spacetimes on which the anisotropies and inhomogeneities have been smeared out. By several different accounts, such conditions are believed to hold during the very early stages of the evolution of the universe. The full grasp of the quantum mechanical system whose classical limit is (10) would help us answer the question whether the homogeneity and isotropy of the universe perceived now is the result of cleverly chosen initial conditions, or the generic endpoint of the dynamics of the spacetime.

Now, the Hamiltonian constraint (10) encodes the tension between the existence of a 
phase of Cosmological Inflation and the notion of naturalness of initial conditions. If we assume that the expansion rate is positive and relatively constant, (10) tells us that $\mathcal{H}_{\text {matter }}$ has to remain also relatively constant during a "long" span of time, essentially a cosmological constant. Although other mechanisms of Inflation exist, the notion of a state with relatively well-defined value for the expansion factor is at odds with a well-defined value of the scale factor, since, as seen above, these quantities are canonically conjugate. We will focus on whether such a state with specific characteristics is actually ordinary in the phase space of General Relativity.

Such definition of "ordinary" really depends on a working definition of quantum gravity, as stressed by [5, 6]. There is also an issue of the global vs. local points of view, in which the two questions "what is the probability of inflation?" and "what is the probability of a sizeable universe have gone through inflation?" have different answers. We will take a local point of view, in which quantum observables are formulated in terms of locally measurable quantitites. Heuristically, one could think of the quantum system as the "microcanonical" in the sense of statistical mechanics, in which there are a multitude of degrees of freedom not accessible macroscopically. The word "microcanonical" is perhaps inappropriate: in truth, there is no "ensemble" to speak of, and the analogy runs better if one thinks of the quantum system as sampling a sizeable portion of the available phase space, as in ergodic theory. The macroscopical, "thermodynamic" system is the one whose phase space is parametrized by $h_{a b}$ and $\pi^{a b}$, that is, General Relativity. Now, a number of proposals for the measure on the

thermodynamic phase space have been proposed [7-10], and the problem of naturalness of inflation has been considered. As such, the final answer relies deeply in regularization one uses to define a measure in the (thermodynamic) phase space. Our proposal is to use the Holographic principle, in the guise of (11), to study the thermodynamic phase space defined by the "first quantized" version of the Hamiltonian constraint (10).

\section{THE WHEELER-DEWITT-FRW EQUATION}

Our objective in this section is to introduce the Wheeler-DeWitt (WDW) equation of the Friedmann-Robertson-Walker (FRW) model to obtain a semiclassical description of the gravitational degrees of freedom. The word semiclassical here means that we are assuming that quantum perturbations of the metric are small compared to fluctuations of the matter 
field. That is what allow us to restrict our considerations only to FRW spacetime, which sets our considerations to classical cosmology.

The WDW equation is obtained simply by casting the canonical quantization prescription into the Hamiltonian constraint of General Relativity. One starts with the 4-D EinsteinHilbert lagrangian coupled to a scalar field $\phi$, especialized to the FRW metric with lapse function $N$ and spatial slices of (normalized) curvature $\kappa$ :

$$
L=-\frac{3}{N} a \dot{a}^{2}+3 N \kappa a+\frac{1}{2 N} a^{3} \dot{\phi}^{2}-N a^{3} V(\phi) .
$$

from which we define the conjugate momenta

$$
p_{a}=-\frac{6}{N} a \dot{a} \quad, \quad p_{\phi}=\frac{1}{N} a^{3} \dot{\phi} \quad
$$

and obtain the hamiltonian

$$
\mathcal{H}=N\left(-\frac{p_{a}^{2}}{12 a}+\frac{p_{\phi}^{2}}{2 a^{3}}+a^{3} V(\phi)-3 \kappa a\right)
$$

The equation of motion for $N$ show us that 13 is actually a constraint given by

$$
H \equiv-\frac{p_{a}^{2}}{12 a}+\frac{p_{\phi}^{2}}{2 a^{3}}+a^{3} V(\phi)-3 \kappa a=0 .
$$

which is just $\mathcal{H}=0$ with $N=1$. It is interesting for our purpose to change the variable $a$ to $\alpha \equiv \log a^{3}$ which is essentially the volume of a patch of comoving volume one. Now, using that

$$
p_{\alpha}=-\frac{2}{3} e^{\alpha} \dot{\alpha}
$$

in equation (14), we have

$$
-\frac{3}{4} p_{\alpha}^{2}+\frac{1}{2} p_{\phi}^{2}+e^{2 \alpha}\left(V(\phi)-3 \kappa e^{-2 \alpha / 3}\right)=0 .
$$

Now, if we impose the canonical quantization rule

$$
p_{\alpha} \rightarrow-i \partial_{\alpha} \quad, \quad p_{\phi} \rightarrow-i \partial_{\phi}
$$


into (15), we obtain a Klein-Gordon-like equation for gravitation given by

$$
\left[\frac{3}{4} \frac{\partial^{2}}{\partial \alpha^{2}}-\frac{1}{2} \frac{\partial^{2}}{\partial \phi^{2}}+e^{2 \alpha}\left(2 V(\phi)-3 \kappa e^{-2 \alpha / 3}\right)\right] \Psi(\alpha, \phi)=0,
$$

which is the Wheeler-DeWitt equation of our model. We can simplify it with the substitution $\alpha \rightarrow \sqrt{3 / 2} \alpha$ giving

$$
\left[\frac{\partial^{2}}{\partial \alpha^{2}}-\frac{\partial^{2}}{\partial \phi^{2}}+2 e^{\sqrt{6} \alpha}\left(V(\phi)-3 \kappa e^{-\sqrt{2 / 3} \alpha}\right)\right] \Psi(\alpha, \phi)=0,
$$

which we shall call the WDW-FRW equation. Note that $\alpha$ plays the role of time translation, or scale-space translation. This will play an important role in the following.

With the WDW-FRW equation we will formulate a semiclassical description of the thermodynamical degrees of freedom of gravitation defined in the configuration space $(\alpha, \phi)$. This "first quantized" version of quantum gravity suffers from all the usual pathologies: i) the spectrum is not bounded from below, which makes the identification of the vacuum very difficult; ii) this problem is exacerbated by the lack of apparent symmetries of the equation - identification of the vacuum state as the one respecting all the symmetries of the equations of motion is hopeless; iii) Without time-invariance, one cannot talk about conservation of the number of quanta, then the need of "second quantization" arises, with all the interpretational problems of creation and annihilation operators of quanta ensuing. We will gleefully overlook all these complications and take (18) as it stands: defining a semiclassical dynamic on the reduced phase-space parametrized by $\alpha, \phi$.

\section{PROBABILITY OF INFLATION}

With all the subtleties disregarded, the system defined by (18) can be thought of as the wave equation in two-dimensional space-time with a complicated, "time-dependent" [11] potential. We will in this section review the calculation of the number of degrees of freedom of the theory as the coincident limit of a suitable Green's function of the system. Such Green's function will satisfy the non-homogeneous equation:

$$
\left[\frac{\partial^{2}}{\partial \alpha^{2}}-\frac{\partial^{2}}{\partial \phi^{2}}+2 e^{\sqrt{6} \alpha}\left(V(\phi)-3 \kappa e^{-\sqrt{2 / 3} \alpha}\right)\right] G\left(\alpha, \phi ; \alpha^{\prime}, \phi^{\prime}\right)=\delta\left(\alpha, \alpha^{\prime}\right) \delta\left(\phi, \phi^{\prime}\right),
$$


which illustrates the ambiguity of such function: not only it is defined up to the addition of a homogeneous term, but also in relativistic theories it defines different Green's functions depending on the prescription of contourning the poles. Moreover, whichever function is picked will have an additional problem: in practice the coincidence limit is divergent and needs to be regularized.

In the following, we will argue that the right choice for Green's function is the Hadamard function, and we shall also review the point-splitting regularization method and show how to uniquely identify the divergent nature of the Hadamard Green's function. Now we turn to the definition of the density of states.

A standard procedure to count states in a quantum theory is conveniently reproduced by analysing the spectral decomposition of the second order hyperbolic equation

$$
\left[\partial_{\alpha}^{2}+\hat{K}(\alpha, \phi)\right] \Psi=0
$$

where $\hat{K}(\alpha, \phi)$ is a second order elliptic differential operator. In particular, for the WDWFRW model,

$$
\hat{K}(\alpha, \phi)=-\partial_{\phi}^{2}+2 e^{\sqrt{6} \alpha}\left(V(\phi)-3 \kappa e^{-\sqrt{2 / 3} \alpha}\right)
$$

Unfortunately, equation (20) is not separable. This makes the analysis of the spectrum quite complicated because one cannot directly Fourier transform the equation, reducing the problem to an eigenvalue equation. However, there is another way to count states by making an analytic continuation of $G_{W}$ and using the Hadamard expansion.

Let us recast (19) in a more convenient way by factoring it with respect to the function $W(\alpha, \phi) / \mu^{2}$

$$
\left(\square_{W}+\mu^{2}\right) G_{W}(\alpha, \phi)=\frac{\delta(\alpha) \delta(\phi)}{\sqrt{g_{W}}},
$$

where $G_{W}$ is the Green function of the WDW-FRW equation, the function $W$ is given by

$$
W(\alpha, \phi)=2 e^{\sqrt{6} \alpha}\left(V(\phi)-3 \kappa e^{-\sqrt{2 / 3} \alpha}\right)
$$

and $\mu$ is intended to be an analogue of the Klein-Gordon mass. In this trick, we are interchanging a description of WDW-FRW in Minkowski flat space with "variable mass" for a 
curved space version with mass $\mu^{2}$ and metric

$$
d s^{2}=\frac{W(\alpha, \phi)}{\mu^{2}}\left(d \alpha^{2}-d \phi^{2}\right)
$$

We will be considering the case where $W>0$, so that the dynamics is dominated by the interaction with matter. In the case where $W<0, \alpha$ and $\phi$ will change roles.

$G_{W}$ is clearly not unique and depends on the boundary conditions, being a fundamental solution of a partial diferential equation. On the other hand, $G_{W}$ presents a singular structure in the coincident limit $\alpha, \phi \rightarrow 0$ which is unique and is called the Hadamard series expansion of $G_{W}\lfloor 12-14]$. The Green's function may be formally written as the inverse operator

$$
G_{W}=\left(\square_{W}+\mu^{2}\right)^{-1}=i \int_{0}^{\infty} d s e^{-i\left(\square_{W}+\mu^{2}\right) s}
$$

were an implicit $\epsilon$-prescription is supposed to define it as a Feynman propagator. Hence, setting $\underline{x} \equiv(\alpha, \phi)$ and $\underline{y} \equiv\left(\alpha^{\prime}, \phi^{\prime}\right)$, we obtain the representation

$$
\begin{aligned}
G_{W}(\underline{x}, \underline{y}) & =i \int_{0}^{\infty} d s\left\langle\underline{x}\left|e^{-i s \square_{W}}\right| \underline{y}\right\rangle e^{-i \mu^{2} s} \\
& \equiv i \int_{0}^{\infty} d s U(s ; \underline{x}, \underline{y}) e^{-i \mu^{2} s}
\end{aligned}
$$

where $U(s ; \underline{x}, \underline{y})$ is the kernel of the associated Schrödinger equation

$$
i \frac{\partial U}{\partial s}=\square_{W} U
$$

In order to solve the initial value problem, $U$ must obey the boundary condition

$$
\lim _{s \rightarrow 0} U(s ; \underline{x}, \underline{y})=\frac{\delta(\underline{x}-\underline{y})}{\sqrt{g_{W}}} .
$$

There is an easy way to see that the coincidence limit of $G_{W}(\underline{x}, \underline{y})$ gives a formula for the number of states. Consider that the Hilbert space of solutions of (22) is labeled by the 
parameter $g$. The number of states is thus given by

$$
\begin{aligned}
\mathcal{N} & \equiv \sum_{g} 1 \\
& =\sum_{g}\left\langle 0 ; g\left|\delta\left(\square_{W}+\mu^{2}\right)\right| 0 ; g\right\rangle \\
& =\frac{1}{\pi} \sum_{g}\left\langle 0 ; g\left|\operatorname{Im}\left(i \int_{0}^{\infty} d s e^{-i\left(\square_{W}+\mu^{2}\right) s}\right)\right| 0 ; g\right\rangle \\
& =\frac{1}{\pi} \sum_{g} \int d^{2} x|W| \operatorname{Im} G_{W}(\underline{x}, \underline{y}) \psi_{g}^{*}(\underline{x}) \psi_{g}(\underline{y}) \\
& =\frac{1}{\pi} \int d x|W| \operatorname{Im} G_{W}(\underline{x}, \underline{x}),
\end{aligned}
$$

where the delta function in the second line must be understood as a projection operator and in the fourth line we used the completeness relation

$$
\sum_{g} \psi_{g}^{*}(\underline{x}) \psi_{g}(\underline{y})=\delta(\underline{x}-\underline{y})
$$

Now to properly define the number of states above we will use the point-splitting method to regularize $G_{W}$.

First we note that eq. (25) sets the singularity structure of $U$ with respect to $s$ and an appropriate Ansatz for a $d$-dimensional manifold in terms of the geodesic distance $\sigma$ is

$$
U(s ; \underline{x}, \underline{y})=\Delta^{1 / 2}(\underline{x}, \underline{y}) \frac{1}{(4 \pi i s)^{d / 2}} e^{i \sigma(\underline{x}, \underline{y}) / 2 s} \sum_{n=0}^{\infty} a_{n}(\underline{x}, \underline{y})(i s)^{n}
$$

which is just the VanVleck-Morette determinant $\Delta^{1 / 2}(\underline{x}, \underline{y})$ times the product of a delta sequence of functions (set to match the delta boundary condition) and a smooth function of $s[14-16]$. Putting this $U$ into (24), we obtain

$$
\begin{aligned}
G_{W}(\underline{x}, \underline{y}) & =-\frac{\Delta^{1 / 2}}{(4 \pi i)^{d / 2}} \int_{0}^{\infty} d s \frac{1}{s^{d / 2}} e^{-i\left(\mu^{2} s-\sigma / 2 s\right)} \sum_{n=0}^{\infty} a_{n}(i s)^{n} \\
& =-\frac{\Delta^{1 / 2}}{(4 \pi i)^{d / 2}} \sum_{n=0}^{\infty} a_{n}\left(-\frac{\partial}{\partial \mu^{2}}\right)^{n} \int_{0}^{\infty} d s \frac{1}{s^{d / 2}} e^{-i\left(\mu^{2} s-\sigma / 2 s\right)}
\end{aligned}
$$

The last equation involves an integral representation of the Hankel function of the second 
kind and may be explicitly written for the case $d=2$ as $[13]$

$$
G_{W}(\underline{x}, \underline{y})=\frac{1}{4} \Delta^{1 / 2} \sum_{n=0}^{\infty} a_{n}\left(-\frac{\partial}{\partial \mu^{2}}\right)^{n} H_{0}^{(2)}\left[\left(-2 \mu^{2} \sigma\right)^{1 / 2}\right] .
$$

Using the resort of an asymptotic series expansion for $H_{0}^{(2)}$, we obtain a series for $G$ and, noting that $G^{(1)}=2 \operatorname{Im} G$, we have [13]

$$
\begin{aligned}
G^{(1)}(\underline{x}, \underline{y}) & =\Delta^{1 / 2}\left\{-L\left[1+\frac{1}{2} \mu^{2} \sigma-\frac{1}{2} a_{1} \sigma+\mathcal{O}\left(\sigma^{2}\right)\right]\right. \\
& +\frac{1}{2}\left[\mu^{-2} a_{1}+\mu^{-4} a_{2}+\mathcal{O}\left(\mu^{-6}\right)\right] \\
& \left.+\frac{1}{4} \sigma\left[2 \mu^{2}-a_{1}-\mu^{-2} a_{2}+\mathcal{O}\left(\mu^{-4}\right)\right]+\mathcal{O}\left(\sigma^{2}\right)\right\}
\end{aligned}
$$

where $L=\gamma+\frac{1}{2} \ln \left|\frac{1}{2} \mu^{2} \sigma\right|$. As our interest remains in the coincidence limit $\sigma=0$ of $G$, we use the fact that the coefficients $a_{n}$ may be calculated quite directly in this limit [16], giving

$$
\begin{aligned}
{\left[\Delta^{1 / 2}\right] } & =1 ; \\
{\left[a_{1}\right] } & =\frac{R}{6} ; \\
{\left[a_{2}\right] } & =\frac{1}{60}\left(R^{2}+2 \square R\right) .
\end{aligned}
$$

As the mass $\mu$ has no physical meaning for us, we set $\mu=1$ and taking the limit $\sigma \rightarrow 0$ we see that the Hadamard series of $G^{(1)}$ has the form

$$
G^{(1)}(\sigma) \sim A \ln \sigma+B
$$

and, hence, presents a logarithm singularity. According to the asymptotic theory of partial differential equations (32) represents the general singular structure of Green's functions of Klein-Gordon equation for $d=2$. We have to find in this divergent expression a measure of the number of degrees of freedom available to the field. Being of the hyperbolic type, the Klein-Gordon equation allows for an infinite number of solutions, but this infinite number comes about because we are including non-physical excitations with arbitrarily small wavelength. We then need a regularization scheme that reflects the physics of the problem; the situation so far not unlike the problem in flat space. We then choose to deal not with the 
terms in the expansion $A$ and $B$ in (32) directly but instead to study their dependence on the factor $W$. Again, the situation is not unlike ordinary quantum field theory in curved spaces where the choice of a vacuum in flat space fixes the relative energy between vacuum in confined spaces (Casimir effect), which are related to the Minkowski vacuum by a conformal transformation. Coming back to (32), the term $A$ has the interpretation of the normalization of the wave-function, so it does not encode the geometric dependence of the degrees of freedom. This leaves us with the term $B$, which should encode the dependence of the number of degrees of freedom. This will be the focus of our attention from now on.

Finally, for the Green's function of eq. (22),$A=-1 / 2 \pi$ and

$$
B=\frac{1}{\pi}\left[-\gamma+\log \sqrt{2}+\frac{R}{12}+\frac{1}{120}\left(R^{2}+2 \square R\right)+\mathcal{O}\left(R^{4}, \square^{2} R\right)\right] .
$$

where $R$ is given by:

$$
R=-\frac{1}{2}\left(\frac{V^{\prime \prime}(\phi)\left(V(\phi)-3 \kappa e^{-\sqrt{2 / 3} \alpha}\right)-\left(V^{\prime}(\phi)\right)^{2}+2 \kappa e^{-\sqrt{2 / 3} \alpha} V(\phi)}{e^{\sqrt{6} \alpha}\left(V(\phi)-3 \kappa e^{-\sqrt{2 / 3} \alpha}\right)^{3}}\right)
$$

One notes that $R$ becomes large in the regime $V(\phi) \approx 3 \kappa a^{-2}$, corresponding to configurations where the potential energy rivals the curvature of the spatial slices. This should be considered a true quantum regime where the semiclassical approximation does not hold. Classically, the quantity $V(\phi)-3 \kappa e^{-\sqrt{2 / 3} \alpha}$ corresponds to the Hubble parameter squared in the FRW equations. We will then consider the regime where this number is positive, in such a way that $R$ is small. Note that this corresponds roughly with the regime where the derivatives of the potential are much smaller than the potential itself, a requisite for slow-roll inflation.

In the semiclassical limit, if the terms of higher order in $R$ are neglected, we have

$$
\mathcal{N}=\frac{1}{2 \pi^{2}} \int d \alpha d \phi|W(\alpha, \phi)|\left[C+\frac{R}{12}\right]
$$

where $C=-\gamma+\log \sqrt{2}$. This is the formal number of states of the Hilbert space of solutions of the WDW-FRW equation, in the point-splitting regularization scheme. The measure is still infinite after an integration over the whole $(\alpha, \phi)$ plane. However, we are mainly interested on the derivative of $\mathcal{N}$, that is, $\mathcal{N}(\alpha)$, which represents the instantaneous number of states for a given size of the universe $\alpha$. It should be noted that the value of $C$ 
changes according to the regularization scheme used. On the other hand, one can try to define a relative number of available states by comparing the expression above with some "fiducial" expression. This can be argued as follows: in the absence of a potential, $R=0$ according to (34). In this situation, one has "pure gravity", and the number of degrees of freedom has to express this fact. Scaling back $\alpha \rightarrow \sqrt{2 / 3} \alpha$ and using the holographic bound to set the range of values allowed for the scalar field:

$$
\mathcal{N}(\alpha)=\frac{3|\kappa|}{\pi^{2}} e^{4 \alpha / 3}|\Delta \phi| \Longrightarrow|\Delta \phi|=K e^{-2 / 3 \alpha}
$$

for some constant $K$. In this manner the number of states associated with pure gravity scales as the area $e^{2 \alpha / 3}$. This can also be seen as a "gravitational uncertainty principle", which states that the spread on the values of the scalar field decreases with the inverse of the square of the scale factor. Large universes would have a small spread of the values of the scalar field, then. One should point out that the interval specified by (35) need not be centered in $\phi=0$. In general there is no symmetry forcing this to be the case. At this point it should be noted that the holographic bound effectively serves as a cutoff on the number of physically available solutions of the Klein-Gordon equation, in the sense that only the number of states given by $\mathcal{N}(\alpha)$ have an interpretation as a geometrical, macroscopic universe. This is successfully incorporated in the quantum theory as a dynamical limit. It is also relevant that this cutoff can take into account anisotropic and dynamical states, as long as they are eigenstates of the scale factor operator $\hat{a}$. In other words, instead of having only one local state labelled with $\alpha$ and $\phi$ in (22), one can have up to $\mathcal{N}(\alpha)$ states associated with these macroscopic quantities. These presumably relating to anisotropic degrees of freedom of these quantities.

Let us digress a little on the curvature terms. We note that, for $\kappa=0$ in (23)

$$
\begin{aligned}
R & =\frac{1}{W} \frac{d^{2}}{d \phi^{2}} \log V(\phi) \\
& =\frac{1}{W} \frac{d}{d \phi}\left(\frac{V^{\prime}}{V}\right) \\
& =\frac{1}{W} \frac{d}{d \phi} \sqrt{2 \epsilon}
\end{aligned}
$$

which allows to relate the Ricci scalar $R$ with the inflationary slow-roll parameter $\epsilon=V^{\prime} / 2 V$. 
Obviously its powers and derivatives are associated with the curvature corrections in (33). If slow-roll inflation is to take place, we then want the curvature corrections to be small.

Therefore, the total number of states for a given size of the universe is then approximately given by the "volume term":

$$
\mathcal{N}(\alpha) \approx \frac{1}{2 \pi^{2}} \int d \phi|W(\alpha, \phi)| \approx \frac{1}{\pi^{2}} e^{2 \alpha} V\left(\phi_{c}\right)|\Delta \phi|
$$

In which we can state the problem: this number is also limited by the holographic bound $\propto e^{2 \alpha / 3}$. Using the result (35), we see that the most probable value of the scalar field $\phi_{c}$ is then veered towards decreasing the value of the potential $V\left(\phi_{c}\right) \propto e^{-2 \alpha / 3}$. This value, however, is incompatible with inflation because of the Hamiltonian constraint: $H^{2} \propto V(\phi)$ induces a linear growth of the scale-factor $a \propto t$, where $H$ is the Hubble parameter.

One can conclude that, by this proposal, slow-roll inflation is supressed by entropic grounds: the volume of phase space of initial conditions of gravity that allow for inflation is just too small for it to be favored dynamically. This conclusions, of course, need not apply to all different models of inflation, which in itself makes for an interesting follow-up to this discussion.

\section{COMMENTS}

The main purpose of this letter is to introduce the concept of the holographic bound as a cutoff for a theory of quantum gravity. The nature of this cutoff is very similar to that of effective "non-renormalizable" field theories: it is a crucial part of the theory, and dictates large-scale behavior, even though the cutoff belongs to high-energy phenomena.

We motivated the employment of the holographic bound seeing it not as a "true bound" on the degrees of freedom, but rather as a limit under which these degrees have geometrical interpretation. The picture that arises is a bit different from usual field theories: rather than a bound on the observables on the system (like the energy), we have a non-local albeit in parameter space - bound that counts all states labelled by a single scale factor. Because of the technical issues, the discussion was kept to spatially homogeneous FRW cosmology coupled to a single scalar field. Using standard procedures to count the total number of states, we could argue that usual slow-roll inflation is entropically supressed with 
this provision.

While the particular conclusion for slow-roll inflation is model dependent, the application of the holographic bound as a cutoff on the number of degrees of freedom in quantum gravity can be used as an universal guideline for studying semiclassical effects in quantum gravity, beyond the Laws governing the temperature of the fields coupled to classical gravity.

The proposal put forward here can be contrasted to the many existent in the literature as being both natural and coordinate independent. The apparent drawback of only be applicable to spherically symmetric situations does not hinder many of the applications, both in cosmology and in black hole dynamics. This point opens an avenue of future developments.

\section{ACKNOWLEDGEMENTS}

We would like to thank Amílcar Queiroz, Luciano Barosi and Francisco Brito for fruitful discussions. One of the authors (FN) acknowledge partial support from CNPq.

[1] G. 't Hooft, "Dimensional reduction in quantum gravity," 1993, gr-qc/9310026. Essay dedicated to Abdus Salam.

[2] W. Fischler and L. Susskind, "Holography and cosmology," 1998, hep-th/9806039.

[3] R. Bousso, "The holographic principle," Rev. Mod. Phys., vol. 74, pp. 825-874, 2002, hep-th/0203101.

[4] R. M. Wald, General Relativity. The University of Chicago Press, 1984.

[5] S. Hollands and R. M. Wald, "Comment on inflation and alternative cosmology," 2002, hep-th/0210001.

[6] S. Hollands and R. M. Wald, "An alternative to inflation," Gen. Rel. Grav., vol. 34, pp. 20432055, 2002, gr-qc/0205058.

[7] S. M. Carroll and H. Tam, "Unitary Evolution and Cosmological Fine-Tuning," July 2010, 1007.1417.

[8] G. W. Gibbons, S. W. Hawking, and J. M. Stewart, "A natural measure on the set of all universes," Nuclear Physics B, vol. 281, no. 3-4, pp. 736 - 751, 1987. 
[9] S. W. Hawking and D. N. Page, "How probable is inflation?," Nuclear Physics B, vol. 298, no. 4, pp. $789-809,1988$.

[10] G. W. Gibbons and N. Turok, "The measure problem in cosmology," Phys. Rev., vol. D77, p. 063516, 2008, hep-th/0609095.

[11] That is, $\alpha$-dependent or $\phi$-dependent, depending on the chosen signature.

[12] J. Hadamard, Lectures on Cauchy's Problem in Linear Partial Differential Equations. New Haven, USA: Yale University Press, 1923.

[13] T. S. Bunch, S. M. Christensen, and S. A. Fulling, "Massive Quantum Field Theory in TwoDimensional Robertson- Walker Space-Time," Phys. Rev., vol. D18, pp. 4435-4459, 1978.

[14] S. A. Fulling, Aspects of Quantum Field Theory in Curved Space-Time, vol. 17. 1989.

[15] B. Dewitt and R. Brehme, "Radiation damping in a gravitational field," Annals of Physics, vol. 9, pp. 220-259, Feb. 1960.

[16] S. M. Christensen, "Regularization, renormalization, and covariant geodesic point separation," Phys. Rev. D, vol. 17, pp. 946-963, Feb 1978. 